\title{
An Information-Theoretic Framework for High-Order Co-clustering of Heterogeneous Objects
}

\author{
Antonio D. Chiaravalloti ${ }^{2}$, Gianluigi Greco ${ }^{1}$, Antonella Guzzo ${ }^{2}$, and Luigi Pontieri ${ }^{2}$ \\ ${ }^{1}$ Dept. of Mathematics, UNICAL, Via P. Bucci 30B, 87036, Rende, Italy \\ 2 ICAR, CNR, Via Pietro Bucci 41C, 87036 Rende, Italy \\ \{ggreco\}@mat.unical.it \\ \{chiaravalloti, guzzo, pontieri\}@icar.cnr.it
}

\begin{abstract}
The high-order co-clustering problem, i.e., the problem of simultaneously clustering several heterogeneous types of domains, is usually faced by minimizing a linear combination of some optimization functions evaluated over pairs of correlated domains, where each weight expresses the reliability/relevance of the associated contingency table. Clearly enough, accurately choosing these weights is crucial to the effectiveness of the co-clustering, and techniques for their automatic tuning are particularly desirable, which are instead missing in the literature. This paper faces this issue by proposing an information-theoretic framework where the co-clustering problem does not need any explicit weighting scheme for combining pairwise objective functions, while a suitable notion of agreement among these functions is exploited. Based on this notion, an algorithm for co-clustering a "star-structured" collection of domains is defined.
\end{abstract}

Keywords: Co-clustering, Mutual Information, Heterogeneous Data.

\section{Introduction}

The problem of clustering heterogeneous objects has become an active research area in recent years. In particular, there is a great deal of literature addressing the clustering of two different types of objects, hereinafter called domains or dimensions, such as documents and terms in text corpora (e.g., [7[4]). This task, usually known as (bidimensional) co-clustering or bi-clustering, has been faced by way of different strategies, including spectral [74] and information-theoretic approaches [5|31].

Some recent works have generalized the bi-clustering problem to the case of more than two domains (short: high-order co-clustering problem) [2 6]8]. In particular, [6] considered a co-clustering problem for "star"-structured domains of the form $D_{X}$, $D_{Y^{1}}, \ldots, D_{Y^{N}}$ (where $N>1$ and $D_{X}$ is the central domain), by defining an objective function $f_{X, Y^{i}}$, for each "auxiliary" domain $D_{Y^{i}}$, whose optimization should lead to the isolation of the best co-clusters over $D_{X}$ and $D_{Y^{i}}$. In order to integrate all such (bi-dimensional) co-clustering subproblems, a linear combination of all pairwise objective functions is optimized, subject to the constraint that consistent clusters are found for the central (shared) domain. More precisely, for each domain $D_{Y^{i}}$, the objective function for co-clustering $D_{X}$ and $D_{Y^{i}}$ is weighted with a factor $\beta_{i}$, such that $\sum_{i=1}^{N} \beta_{i}=1$. Clearly, extending this setting to arbitrary pairwise interactions mainly requires to equip with a weight, say $\beta_{A, B}$, each pair of (arbitrarily) correlated domains $A$ and $B$ (cf. [2]). 

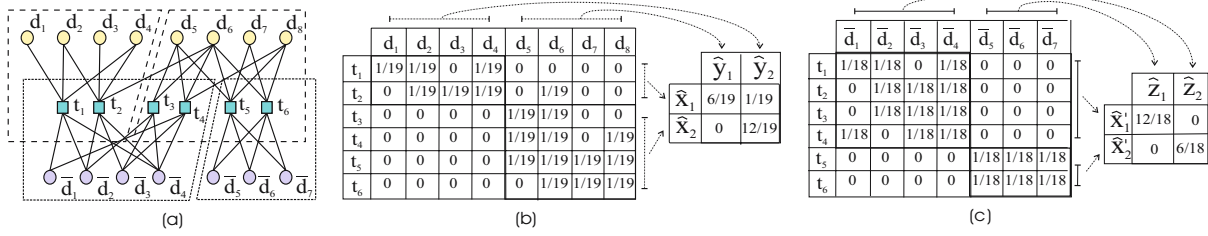

Fig. 1. Co-clustering a text corpus: (a) Spectral and (b-c) Information-theoretic approach

For example, Fig. 3(a) reports a chart (discussed in Section 4) that evidences the quality of the clustering (measured via the loss in mutual information occurring when replacing the original domains with their clustered versions) at the varying of $\beta_{1}$, over two syntectic datasets having two auxiliary domains. It is easy to see that the best (cf. minimum) value for the loss of mutual information in the first domain is achieved when $\beta_{1}=1$, i.e., when the clustering is performed along this domain only. Similarly, the best value for the second domain is achieved when $\beta_{1}=0$ (and $\beta_{2}=1$ ). Thus, setting the values for the weights strongly affects the quality of the results over the co-clustering.

In general, there may be not enough knowledge on the reliability/relevance of pairwise correlation data to set the weights precisely. Hence, some method for their automatic tuning should be defined, as already stated in [6]. In fact, we mainly aim at facing such an open issue, for the specific case of star-structured domains.

In more details, in Section 2, we introduce an information-theoretic framework which generalizes that in [5] and allows to co-cluster an arbitrary number of domains forming a star structure. Notably, this setting fits a wide range of relevant real-world data, like those describing relationships among authors, conferences, papers and keywords in academic publications (with publications constituting the central domain).

In order to address such a problem without using any arbitrary weighting scheme, in Section 3, we propose and study an algorithm that solves the High-Order Co-Clustering by computing Agreements for contrasting Domain objective functions (short: $\mathrm{AD}-\mathrm{HOCC}$ algorithm). The basic idea is to consider a notion of agreement, such that a clustering of the central domain is found which guarantees that each partial objective function is not "too far" from its optimal value.

Results from test on both synthetic and real data are finally illustrated in Section 4.

\section{Formal Framework}

Let $D_{X}=\left\{x_{1}, \ldots, x_{m}\right\}, D_{Y^{1}}=\left\{y_{1}^{1}, \ldots, y_{n}^{1}\right\}, \ldots, D_{Y^{N}}=\left\{y_{1}^{N}, \ldots, y_{n}^{N}\right\}$ be $N+1$ domains, i.e., sets of values, and let $X, Y^{1}, \ldots, Y^{N}$ be discrete random variables taking values in $D_{X}, D_{Y^{1}}, \ldots, D_{Y^{n}}$, resp. The domains are assumed to form a star structure, i.e., the auxiliary domains $D_{Y^{i}}$, for $i=1 . . n$ are pairwise independent, while each of them is correlated with the central domain $D_{X}$. Let then $p_{i}\left(X, Y^{i}\right)$, with $1 \leq i \leq$ $N$, denote the joint probability distribution between $X$ and $Y^{i}$, i.e., $p_{i}\left(x, y^{i}\right)$ is the probability that $X$ takes the value $x \in D_{X}$ and $Y^{i}$ takes the value $y^{i} \in D_{Y^{i}}$.

Assume that the values of $D_{X}$ are to be clustered into $k$ clusters, say $\widehat{D}_{X}=\left\{\widehat{x}_{1}\right.$, $\left.\widehat{x}_{2}, \ldots, \widehat{x}_{k}\right\}$, and those of $D_{Y^{i}}$ in $l_{i}$ clusters, say $\widehat{D}_{Y^{i}}=\left\{\widehat{y}_{1}, \widehat{y}_{2}, \ldots, \widehat{y}_{l_{i}}\right\}$, for each $i$ 
in 1..N. Then, a high-order co-clustering for $Y^{1}, \ldots, Y^{N}$ w.r.t. $X$ is a tuple $C=$ $\left\langle C_{X}, C_{Y^{1}}, \ldots, C_{Y^{N}}\right\rangle$, such that $C_{X}: D_{X} \mapsto \widehat{D}_{X}$, and $C_{Y}: D_{Y^{i}} \mapsto \widehat{D}_{Y^{i}}$, for $i=1 \ldots N$. For brevity, for each random variable $W$ and its associated domain $D_{W}$, the set of all possible mappings from $D_{W}$ to its clusters is denoted by $\mathcal{P}(W)$. Moreover $\widehat{W}_{C_{W}}=C_{W}(W)$ is the random variable denoting the cluster assigned to $W$, through the function $C_{W}$, defined on $D_{W}$ and ranging over $\mathcal{P}(W)$. Like in [5], we use lowercase letters for domain elements, and upper-case letters for the random variable ranging over them; in addition, hatted letters are reserved for clusters, and clustered random variables. Also, $\widehat{W}_{C_{W}}$ will be shortened as $\widehat{W}$ whenever $C_{W}$ is clear from the context.

Example 1. Let $\mathrm{d}_{1}, \ldots, \mathrm{d}_{6}$ be documents (e.g., academic papers), and $\mathrm{t}_{1}, \ldots, \mathrm{t}_{8}$ be terms. In Fig. 1.(a) the occurrences of terms in documents are represented as edges, while terms and documents are depicted as nodes (of two different types). The problem can be easily modelled in an information-theoretic framework by defining $X$ and $Y$ to be two random variables taking values in $\left\{\mathrm{d}_{1}, \ldots, \mathrm{d}_{6}\right\}$ and $\left\{\mathrm{t}_{1}, \ldots, \mathrm{t}_{8}\right\}$, respectively. Let $p(X, Y)$ be the joint probability distribution between $X$ and $Y$ represented in a tabular form in Fig. 1 b) 1 E.g., $p\left(\mathrm{~d}_{1}, \mathrm{t}_{2}\right)=\frac{1}{19}$ is the frequency of the event of having $\mathrm{t}_{2}$ occur in document $\mathrm{d}_{1}$, while $p\left(\mathrm{~d}_{1}, \mathrm{t}_{3}\right)=0$ indicates that $\mathrm{t}_{3}$ does not occur in $\mathrm{d}_{1}$. In this setting we can consider the problem of co-clustering both documents and terms in two clusters, say $\left\{\widehat{x}_{1}, \widehat{x}_{2}\right\}$ and, resp., $\left\{\widehat{y}_{1}, \widehat{y}_{2}\right\}$. An example co-clustering $\left\langle C_{X}, C_{Y}\right\rangle$ is shown in the same figure, where $C_{X}$ is the function mapping $\mathrm{d}_{1}$ and $\mathrm{d}_{2}$ to $\widehat{x}_{1}$ and every other document to $\widehat{x}_{2}$, while $C_{Y}$ maps $t_{1}, \ldots, \mathrm{t}_{4}$ to $\widehat{y}_{1}$ and all the other terms to $\widehat{y}_{2}$.

Assume that some information is available on document authors, say $a_{1}, \ldots, a_{7}$. Based on authorship data, reported again in Fig. 1 a), we can consider the problem of coclustering all of the three domains. This is just a high-order co-clustering problem over star-structured domains, which essentially amounts to finding a tuple $\left\langle C_{X}, C_{Y}, C_{Z}\right\rangle$, where $Z$ is a random variable taking values in $\left\{a_{1}, \ldots, a_{7}\right\}$. If one looks at authors independently of terms, a natural co-clustering is $\left\langle C_{X}^{\prime}, C_{Z}\right\rangle$, where $C_{X}^{\prime}$ is the function mapping $\mathrm{d}_{1}, \ldots, \mathrm{d}_{4}$ to $\widehat{x}_{1}$ and every other document to $\widehat{x}_{2}$, and $C_{Z}$ is the function mapping $a_{1}, \ldots, a_{4}$ to $\widehat{z}_{1}$ and every other author to $\widehat{z}_{2}-$ see Fig. 11 c).

As the effect of co-clustering can be viewed as a sort of information compression, the co-clustering problem can be turned in the search for a fixed-size compression scheme preserving as much as possible of the original mutual information. To this aim, for any auxiliary domain $D_{Y^{i}}$, one can compute the mutual information $I\left(X ; Y^{i}\right)$ between the random variables $X$ and $Y^{i}$, ranging over $D_{X}$ and $D_{Y^{i}}$, respectively. Then, the quality of a multi-dimensional co-clustering can be assessed by taking into account the loss of mutual information that occurs when replacing the original variables $X, Y^{1}, \ldots, Y^{N}$ with their clustered versions $\widehat{X}_{C_{X}}, \widehat{Y}^{1} C_{Y^{1}}, \widehat{Y}^{N} C_{Y^{N}}$. Hereinafter, for brevity, the loss of mutual information pertaining the $i$-th auxiliary dimension will be denoted by $\Delta I_{i}\left(C_{X}, C_{Y^{i}}\right)=I\left(X ; Y^{i}\right)-I\left(\widehat{X}_{C_{X}} ; \widehat{Y}^{i} C_{Y^{i}}\right)$ (or, shortly, $\left.\Delta I_{i}\right)$.

For the base case of $N=1$, an algorithm that computes a (locally) optimal coclustering has been presented in [5], where it was shown that the mutual information

\footnotetext{
${ }^{1}$ For the sake of exposition, the joint distribution shown here just results from normalizing a binary association matrix between terms and documents.
} 
loss caused by clustering $X$ and $Y^{i}$ can be expressed as a dissimilarity between the original joint distribution $p_{i}(\cdot, \cdot)$ and a function $q_{i}(\cdot, \cdot)$ that approximates it. More precisely: $\Delta I_{i}=\mathcal{D}\left(p_{i}\left(X, Y^{i}\right) \| q_{i}\left(X, Y^{i}\right)\right)$, where $\mathcal{D}(\cdot \| \cdot)$ denotes the Kullback-Leibler (KL) divergence, and $q_{i}\left(X, Y^{i}\right)$ is a function, preserving all marginals of $p_{i}$, of the form $q_{i}(X, Y)=p_{i}\left(\widehat{X}, \widehat{Y}^{i}\right) \cdot p_{i}(X \mid \widehat{X}) \cdot p_{i}\left(Y \mid \widehat{Y}^{i}\right)$.

Thus, the pairwise co-clustering problem can be solved by searching for the function $q_{i}$ that is most similar to $p_{i}$, according to $\mathcal{D}$. To this purpose, an alternate minimization scheme is used in [5] which considers only one dimension per time. In the rest of the paper, we investigate the case of $N>1$, and define a co-clustering approach that ensures as low as possible values for all the information loss functions $\Delta I_{i}$.

\section{Agreement Method}

A major problem occurring while optimizing the losses of mutual information $\Delta I_{i}$, for each $1 \leq i \leq N$, is that the best co-clustering for $D_{X}$ and $D_{Y^{i}}$ may not comply with the best co-clustering for $D_{X}$ and $D_{Y^{j}}$ for $j \neq i$, over the values in domain $D_{X}$.

Example 2. Consider again the data set in Fig. 1. While co-clustering along each auxiliary dimensions independently, the co-clusterings $\left\langle C_{X}, C_{Y}\right\rangle$ and $\left\langle C_{X}^{\prime}, C_{Z}\right\rangle$ introduced in Example1 1 seem to be very good candidate solutions. This should be also evident by looking at the graphical representation in Fig. 1.a), where these clusters in fact induce some "optimal" cut over the nodes. However, these two optimal bi-clusterings do not conform with each other, since $C_{X} \neq C_{X}^{\prime}$ and, therefore, there is no immediate way for extending them into a global high-order co-clustering.

As discussed previously, a way for jointly optimizing the losses of mutual information $\Delta I_{i}$, for each $1 \leq i \leq N$, is to linearly combine these individual functions into a global one. Thus, one can try to minimize the quantity $\sum_{i=1}^{N} \beta_{i} \cdot \Delta I_{i}$, where $\beta_{1}, \ldots, \beta_{N}$ are suitable weights such that $\sum_{i=1}^{N} \beta_{i}=1$.

Since, in general, there may be no knowledge enough to set the coefficients in a precise manner, we abandon the idea of using a pre-fixed weighting scheme and restate the co-clustering problem as a multi-objective optimization of all functions $\Delta I_{i}=I\left(X ; Y^{i}\right)-I\left(\widehat{X} ; \hat{Y}^{i}\right)$.

Moreover, it may well be the case that no clustering function $C_{X}$ exists over the values of the central domain that allows to achieve a minimal information loss over all the other dimensions - this is, e.g., the case of the clusters in Fig. 11 Therefore, we introduce a notion of agreement to represent a sort of optimal "compromise" among the different (and potentially discordant) goals, which are autonomously pursued by all the bi-dimensional co-clustering subproblems.

Actually, it can be shown that computing an optimal agreement is NP-hard. Indeed, it requires the computation of the optimal clustering over each dimension alone, which is NP-hard of its own. Accordingly, the following definition states, in a pragmatic way, the notion of agreement under a "local" perspective, only.

Definition 1. A high-order co-clustering $C=\left\langle C_{X}, C_{Y^{1}}, \ldots, C_{Y^{N}}\right\rangle$ is said to be an $\alpha$-agreement for $Y^{1}, \ldots, Y^{N}$ w.r.t. $X$ if, for each $Y^{i}$ with $1 \leq i \leq N$, the following conditions hold: 


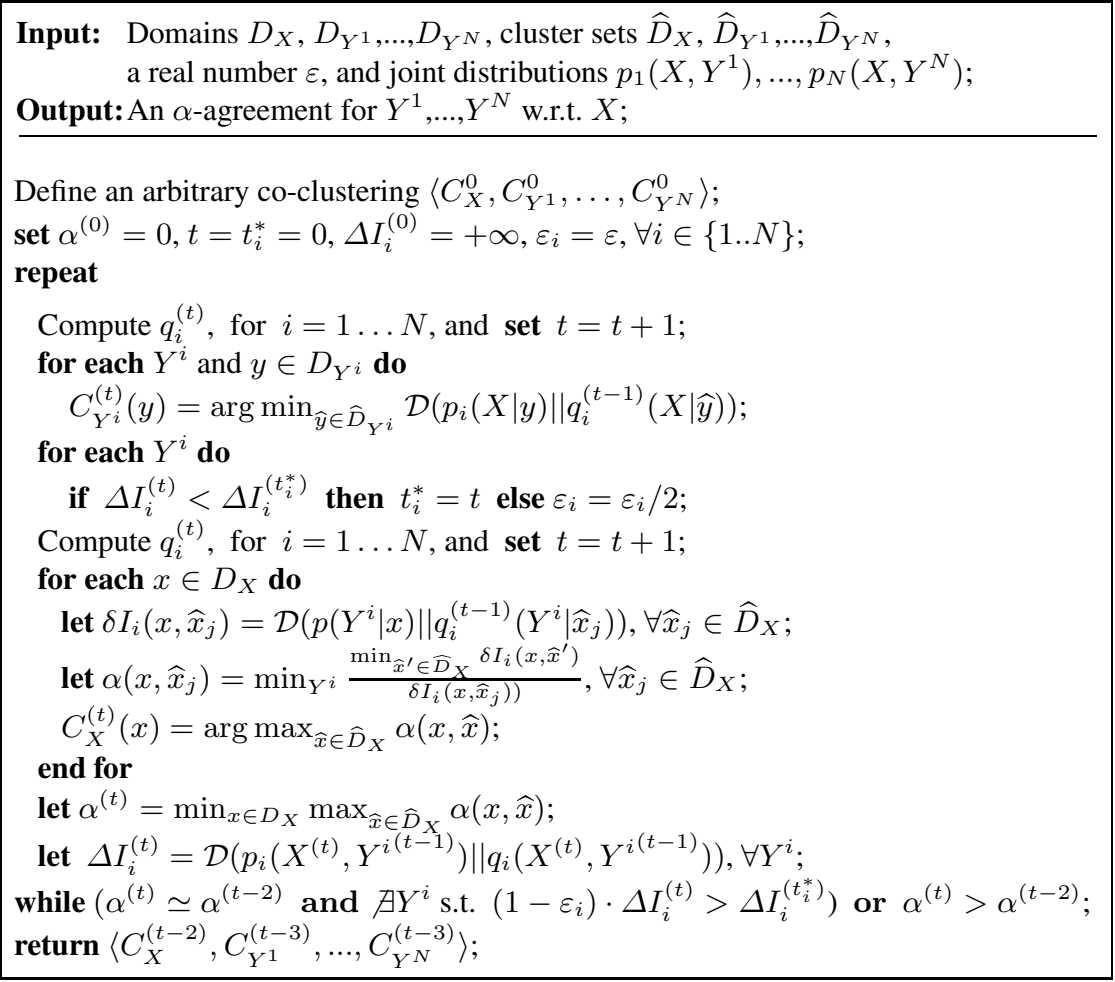

Fig. 2. Algorithm $A D-H O C C$

(a) $\forall C^{\prime} \in \mathcal{P}\left(Y^{i}\right), \Delta I_{i}\left(C_{X}, C^{\prime}\right) \geq \Delta I_{i}\left(C_{X}, C_{Y^{i}}\right)$, and

(b) $\forall C^{\prime \prime} \in \mathcal{P}(X), \Delta I_{i}\left(C^{\prime \prime}, C_{Y^{i}}\right) \geq \alpha \times\left[\Delta I_{i}\left(C_{X}, C_{Y^{i}}\right)\right]$.

If there exists no $\alpha^{\prime}>\alpha$ satisfying condition (b), the agreement is said maximal.

Notably, $\alpha$ is a sort of quality measure assessing the ability of approximating some local optimum of each function $\Delta I_{i}$. As an extreme case, when $\alpha=1$ and $N=1$, an $\alpha$-agreement is a local optimum for the two-dimensional co-clustering problem.

Algorithm $A D-H O C C$. In Fig. 2 an algorithm, named $A D-H O C C$, is shown that computes a maximal $\alpha$-agreement, based on a local, alternate, optimization scheme. First an initial arbitrary co-clustering $\left\langle C_{X}^{0}, C_{Y^{1}}^{0}, \ldots, C_{Y^{N}}^{0}\right\rangle$ is computed, which is eventually refined in the main loop.

At each repetition $t$, the optimal clustering $C_{Y^{i}}^{(t)}$ is computed for each auxiliary domain $Y^{i}(1 \leq i \leq N)$, based on the current clustering for the central domain $X$. Intuitively, this is carried out with the aim of assigning $y$ to the cluster in $\widehat{D}_{Y^{i}}$ leading to the minimization of the loss of mutual information. Subsequently, the iteration continues by computing the optimal clustering $C_{X}^{(t)}$ for the central domain, based on the just computed clusterings for the auxiliary domains. This step is crucial for getting an agreement and is, thus, discussed in more details below. 
Prelimiary, for each value $x \in D_{X}$ and each cluster $\widehat{x}_{j} \in \widehat{D}_{X}$, we compute the contribution $\delta I_{i}\left(x, \widehat{x}_{j}\right)$ that would be given to the information loss over each domain $Y^{i}$ by assigning $x$ to $\widehat{x}_{j}$. All these values are normalized w.r.t. the best possible assignment for $x$, i.e., $\min _{\widehat{x}^{\prime} \in \widehat{D}_{X}} \delta I_{i}\left(x, \widehat{x}^{\prime}\right)$, and the worst possible mapping $\alpha\left(x, \widehat{x}_{j}\right)$ is computed.

The algorithm eventually chooses the best over such worst mappings, i.e., an element $x$ is mapped to the cluster $\widehat{x} \in \widehat{D}_{X}$ maximizing the value $\alpha(x, \widehat{x})$, according to the formula:

$$
C_{X}^{(t)}(x)=\arg \max _{\widehat{x} \in \widehat{D}_{X}} \alpha(x, \widehat{x})
$$

In addition, the value for $\alpha^{(t)}$ characterizing the guarantee for the agrement is computed according to the formula: $\alpha^{(t)}=\min _{x \in D_{X}} \max _{\widehat{x} \in \widehat{D}_{X}} \alpha(x, \widehat{x})$.

The algorithm keeps on iterating till the value $\alpha^{(t)}$ increases, i.e., as long as a better agreement is discovered. Actually, it tolerates that some bi-clustering objective function temporarily get worse, provided that it remains close enough to its best value found so far. To this aim a real number $\varepsilon$ is also required in input to denote the range of tolerance admitted. Such a behavior is meant to reduce the risk of underestimating some partial optimum, as the strategy adopted here does not guarantee that every objective function monotonically decreases. Notice, however, that the width of these tolerance ranges is progressively reduced during the search, thus ensuring termination.

Discussion and comparison with related work. Let $I$ be the number of iterations, $N$ be the number of auxiliary domains, and $M$ be is upper bound for both the size of any domain and the number of nonzero elements in any joint distribution matrix.

It is easy to see that algorithm $A D-H O C C$ converges, at a step $t=O(N \cdot M$. $\left.\left(k+\sum_{i} l_{i}\right) \cdot I\right)$, to a high-order co-clustering for $Y^{1}, \ldots, Y^{N}$ w.r.t. $X$ such that $\forall C_{X}^{\prime} \in$ $\mathcal{P}(X), C_{Y^{1}}^{\prime} \in \mathcal{P}\left(Y^{1}\right), \ldots, C_{Y^{N}}^{\prime} \in \mathcal{P}\left(Y^{N}\right)$, and for each $i$ in $1 . . N$ :

(a) $\Delta I_{i}\left(C_{X}^{(t-2)}, C_{Y^{i}}^{\prime}\right) \geq \Delta I_{i}\left(C_{X}^{(t-2)}, C_{Y^{i}}^{(t-3)}\right)$, and

(b) $\Delta I_{i}\left(C_{X}^{\prime}, C_{Y^{i}}^{(t-3)}\right) \geq \alpha^{(t)} \Delta I_{i}\left(C_{X}^{(t-2)}, C_{Y^{i}}^{(t-3)}\right)$.

Moreover, there is no $\bar{\alpha}>\alpha^{t}$ satisfying condition (b).

Note that when applied over domains $D_{X}, D_{Y^{1}}, \ldots, D_{Y^{N}}$, algorithm AD-HOCC does not, in general, guarantee that all pairwise mutual information losses monotonically decrease. Yet, at each step $t \geq 2$, the decrease in the mutual information $I_{i}$ along each dimension $Y^{i}$ is bounded: $\alpha^{(t)} \times \Delta I_{i}^{(t)} \leq \Delta I_{i}^{(t-2)}$.

As discussed above, previous approaches to the high-order co-clustering problem [26] score individual objective functions via some weights without discussing the problem of their automatic tuning. Besides addressing this issue, algorithm $\mathrm{AD}-\mathrm{HOCC}$ overcomes some limitations of the approach in [6]. For instance, $\mathrm{AD}-\mathrm{HOCC}$ can divide the domains in any arbitrary number of clusters, while the approach in [6] was explicitly developed for the bi-partite co-clustering problem, only.

Notably, the algorithm in [2] has been designed for general pairwise relationships, and can automatically select the number of cluster for each domain, based on a schema that interleaves top-down clustering of some domains and bottom-up clustering of the others, along with a local correction routine. However, this generality comes with a cost: the algorithm in [2] runs in $O\left(\max _{W}\left\{\log \left|\widehat{D}_{W}\right|, \log \left(\left|D_{W}\right| /\left|\widehat{D}_{W}\right|\right)\right\} \cdot \max _{W}\left\{\left|D_{W}\right|^{3}\right\}\right)$, 


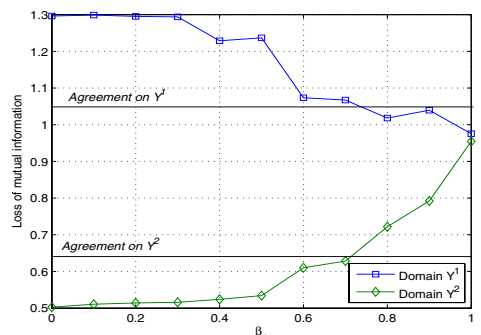

(a)

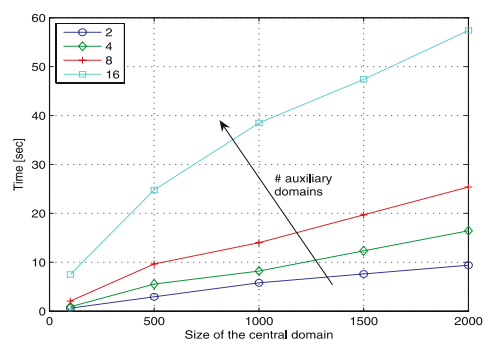

(b)

Fig. 3. Loss of mutual information (a) and computation time (b) on synthetic data

where $W$ denotes any input domain, while a quadratic dependence on $D_{W}$ is ensured only when two domains are to be co-clustered.

\section{Experiments}

This section provides an empirical study of the proposed technique, based on experiments over both synthetic and real data, which were performed by running a Java implementation of the algorithm in Section 3 on a 1600MHz/512MB Pentium IV machine equipped with Windows XP Professional. In order to reduce the statistical bias due to the choice of initial clusterings, every measure has been averaged over 10 runs.

Synthetic data have been produced through an ad-hoc Java generator with the following parameters: $(i)$ the number $N>1$ of auxiliary domains, (ii) the size of the domains $D_{X}, D_{Y^{1}}, \ldots, D_{Y^{N}}$, (iii) the number of required clusters along each domain, (iv) a noise factor $\theta$, and ( $v$ ) a "disagreement" factor $\gamma$. Roughly speaking, the latter basically expresses the maximum percentage of values in $D_{X}$ that would be assigned to different clusters when considering two different contingency tables.

A first series of experiments have been conducted to asses the behavior of the $\mathrm{AD}-\mathrm{HOCC}$ algorithm w.r.t. a "prototypical" co-clustering method that optimizes a linear combination of the information losses, with weights $\beta_{i}$. To this aim, a linear combination algorithm has been implemented by modifying the way $\mathrm{AD}-\mathrm{HOCC}$ selects the cluster $\widehat{x} \in \widehat{D}_{X}$ to which each element has to be assigned. Indeed, rather than using the strategy in Equation 1 the optimal clustering $C_{X}^{(t)}$ is computed as $C_{X}^{(t)}(x)=$ $\arg \min _{\widehat{x} \in \widehat{D}_{X}} \sum_{i=1}^{N} \beta_{i} \cdot \mathcal{D}\left(p_{i}\left(Y^{i} \mid x\right) \| q_{i}^{(t-1)}\left(Y^{i} \mid \widehat{x}\right)\right)$.

Fig. 3. a) shows the mutual information loss at the varying of $\beta_{1}\left(\beta_{2}=1-\beta_{1}\right)$, for a dataset with 2 auxiliary domains, which was built by using a fixed size (1000) and a fixed number of clusters (2) for every domain, and by setting $\gamma=\theta=0.2$. Note that, for each domain, the information loss produced by $\mathrm{AD}-\mathrm{HOCC}$ is always ("slightly") higher than the one found by the linear-combination approach when it just considers that domain (i.e., when $\beta_{1}=1$ for $Y^{1}$, and $\beta_{1}=0$ for $Y^{2}$ ).

In addition, we computed the time spent against several data sets, all having a fixed size of 100 for auxiliary domains, and generated with $\gamma=0.1$ and $\theta=0.05$. Actually, different values have been considered for the size of the central domain (up to 2000 


\begin{tabular}{|c||c|c|c|}
\hline & agr. & $\beta_{t}=1$ & $\beta_{t}=0$ \\
\hline \hline lokay-m/williams-w3 & 0.82 & 0.62 & 0.62 \\
\hline kitchen-l/sanders- $r$ & 0.77 & 0.75 & 0.76 \\
\hline
\end{tabular}

Fig. 4. Mutual information loss and micro-averaged precision on real data

values) and for the total number of domains (up to 16). Results shown in Fig. 3(b), confirm the linear dependence of the computation time on both parameters (cf. Section 3).

In order to validate the proposed approach against real data, we selected two directories, namely lokay- $m$ and williams-w3, from the preprocessed email datasets available at http://www.cs.umass.edu/ ronb/enron_dataset.html. The resulting data set consists of 275 documents organized in 11 and 18 sub-folders, respectively. Then, the document-byterm and the document-by-category matrix have been built, where categories correspond to the sub-folders. Totally, 1074 terms were selected.

Precision results obtained when partitioning the emails into two clusters are shown in Fig. 4. More specifically, we report the standard micro-averaged precision measures (also used, e.g., in [2]) computed by assuming that the main directories lokay-m and williams-w3 represent "ground truth" classes for the emails. The table also shows results for similar experiments conducted over directories kitchen-l and sanders-r. Notice that, in both cases, $\mathrm{AD}-\mathrm{HOCC}$ is able to find an accurate solution corresponding to some intermediate value of $\beta_{t}$. Indeed, the precision with $\mathrm{AD}-\mathrm{HOCC}$ is superior to the one the linear combination achieves in both the extreme scenarios for $\beta_{t}$.

\section{References}

1. A. Banerjee, I. Dhillon, J. Ghosh, S. Merugu, and D. S. Modha. A generalized maximum entropy approach to bregman co-clustering and matrix approximation. In Proc. 10th ACM SIGKDD Conf. on Knowledge Discovery and Data Mining (KDD04), 509-514, 2004.

2. R. Bekkerman, R. El-Yaniv, and A. McCallum. Multi-way distributional clustering via pairwise interactions. In Proc. 22nd Intl. COnf. on Machine Learning (ICML05), 41-48, 2005.

3. P. Berkhin and J. D. Becher. Learning simple relations: Theory and applications. In Proc. 2nd SIAM Conf. on Data Mining (SDM02), 2002.

4. I. S. Dhillon. Co-clustering documents and words using bipartite spectral graph partitioning. In Proc. 7th ACM SIGKDD Conf. on Knowledge Discovery and Data Mining (KDD01), 269274, 2001.

5. I. S. Dhillon, S. Mallela, and D. S. Modha. Information-theoretic co-clustering. In Proc. 9th ACM SIGKDD Conf. on Knowledge Discovery and Data Mining (KDD03), 89-98, 2003.

6. B. Gao, T.Y. Liu, X. Zheng, Q.-S. Cheng, and W.-Y. Ma. Consistent bipartite graph copartitioning for star-structured high-order heterogeneous data co-clustering. In Proc. 11th ACM SIGKDD Conf. on Knowledge Discovery and Data mining (KDD05)), 41-50, 2005.

7. H. Zha, X. He, C. Ding, H. Simon, and M. Gu. Bipartite graph partitioning and data clustering. In Proc. 10th ACM Intl. Conf. on Information and Knowledge Management (CIKM01), 25-32, 2001.

8. L. Zhao and M.J. Zaki. TRICLUSTER: an effective algorithm for mining coherent clusters in 3D microarray data. In Proc. of the 2005 ACM SIGMOD Conf. on Management of Data, 694-705, 2005. 